\title{
Adults with severe Japanese encephalitis: a retrospective analysis of 9 cases in Linyi, China
}

\author{
Qi Su ${ }^{1,2} \cdot$ Zhong-Xiang $\mathrm{Xie}^{2} \cdot$ Feng He ${ }^{2} \cdot$ Zhen-Chuan $\mathrm{Liu}^{2} \cdot$ Xiao-Jie Song ${ }^{2} \cdot \mathrm{Fu}^{2}$-Chun Zhao ${ }^{2} \cdot{\text { Dibin } \mathrm{Li}^{3}}^{3}$ \\ Feng-Yuan $\mathrm{Che}^{2}$ (1)
}

Received: 23 July 2020 / Accepted: 27 October 2020 / Published online: 9 November 2020

(C) Fondazione Società Italiana di Neurologia 2020

\begin{abstract}
Objective Japanese encephalitis (JE) is a critical problem of public health worldwide; however, there is limited data about the clinical features and indicators of outcome in adults with severe Japanese encephalitis.

Methods The clinical manifestations and laboratory study on brain neuroimaging of patients with severe JE were statistically analyzed retrospectively. All patients were followed up for 6 months after discharge. The patients were grouped into good outcome and poor outcome according to the results of the follow-up.

Results This retrospective study consists of 9 adults with severe JE, including 5 cases with poor outcome, defined as the modified Rankin Scale (mRS) scores of greater than or equal to 4 points, and remained ventilator dependent. Typical clinical manifestations of JE include fever (100\%), altered consciousness (100\%), headache (66.7\%), flaccid weakness $(66.7 \%)$, and status epilepticus (44.4\%). Serological examination revealed that a higher percentage of neutrophils and a lower percentage of lymphocytes at admission may be associated with a poor outcome. Abnormal neuroimaging of the thalamus (85.7\%), hippocampal (71.4\%), midbrain (28.6\%), and basal ganglia (14.3\%) was found. $42.9 \%$ of patients left severe irreversible disability, and the most prominent were mental symptoms (71.4\%) and memory or understanding disorder $(57.1 \%)$.

Conclusion Our data suggest that respiratory failure is one of the important causes of early death. Serologic examination, coma, and status epilepticus may indicate a poor outcome for severe JE. Additionally, the hippocampus is the second most common lesion in the adults with severe JE. A large-scale clinical trial is required to further confirm these conclusions.
\end{abstract}

Keywords Japanese encephalitis $\cdot$ Outcome $\cdot$ Clinical manifestations $\cdot$ Neurological features $\cdot$ Linyi

Qi Su and Zhong-Xiang Xie contributed equally to this work.

Supplementary Information The online version contains supplementary material available at https://doi.org/10.1007/s10072-02004867-8.

Dibin Li

lidibin111@163.com

$\triangle$ Feng-Yuan Che Chefy2020@163.com

1 School of Clinical Medicine, Weifang Medical University, Weifang 261042, Shandong, China

2 Department of Neurology, Linyi People's Hospital, Wuhan Road, Lanshan District, Linyi 276003, Shandong, China

3 Department of Neurology, Emergency Medical Center, Beijing Chaoyang Integrative Medicine, Beijing 100020, China

\section{Introduction}

Japanese encephalitis (JE) is an acute infectious disease, a mosquito-borne viral infection of the CNS. Japanese encephalitis virus (JEV) enters into the CNS via the blood-brain barrier (BBB), causing intense inflammation and neuron death [1]. The incidence of JE shows obvious seasonal features and is characterized by rapid onset and high mortality, which is clinically manifested by high fever, headache, disturbance of consciousness, epilepsy, and limb paralysis [2]. According to the World Health Organization, JEV is a main cause of severe encephalitis in Asia with 70,000 cases every year, epidemic in north and endemic in south [3]; approximately $20-30 \%$ of patients die and $30 \sim 50 \%$ of the JE survivors will have severe neurological deficits or mental sequelae [4]. Annual incidence of JE cases varies by viral mutation, endemic regions, and age [5]; with the rise of the vaccination rate of JE, the incidence 
gradually decreased; however, adult outbreaks have begun to appear in some areas in recent years and the specific causes are not clear yet. Predictors of poor outcome for children have been reported that is characterized by high fever, deep coma, a short prodromal stage, dyspnea, decerebration, and neurological dysfunctions [6]. However, adults with severe JE may manifest a variety of life-threatening symptoms, and there are limited studies on it and prognostic indicators. In this study, we analyzed and summarized the clinical data, laboratory and imaging findings and other factors that may affect outcomes of 9 adults with severe JE at a single center in Linyi, China, from July 2017 to August 2018, to enrich and expand the current clinical knowledge of severe JE and clinical knowledge after the acute stage of JE.

\section{Subjects and methods}

\section{Participants, follow-up, and subgroups}

Nine adults with severe JE were admitted to Linyi People's Hospital, China, from July 2017 to August 2018 for analysis. All cases have the history of neither vaccination nor travel to JE outbreak areas. They all developed symptoms in July and August, the time with activated mosquito. Informed consent was obtained from each patient. The diagnosis of JE was based on the following criteria: (1) Fundamental condition: Patients presenting with acute encephalitis syndrome (AES), characterized by acute onset of high fever, altered mental status, and/or new onset of seizures[7], complicated with coma, status epilepticus, upper GI hemorrhage, decerebrate rigidity, pulmonary edema, or peripheral vascular failure. (2) Support condition: (I) The patient has traveled or lived from an endemic area of JE. (II) Elisa showed positive IgM antibody for JEV in serum or brain crest fluid. (III) CT and MRI of the brain showed lesions in the thalamus, hippocampus, basal ganglia, midbrain, and other common site of JE. Patients with severe JE should meet the fundamental conditions and two or more support conditions [8].

The detailed records of all patients were collected, including sex, age, current history, past history, personal history, and family history. A complete physical examination of the nervous system was carried out, and the changes of condition before and after admission were recorded. The clinical manifestations and signs were observed in patients with fever, headache, vomiting, quadriplegia, consciousness disorder, epilepsy, dyspnea, hypermyotonia, meningeal irritation, and pathology.

All patients were followed up once every month for consecutive 6 months. A functional outcome score (modified Rankin Scale, mRS) was performed to assess the recovery after 6 months. According to mRS score, patients with a score less than 4 (no sequelae, mild, or moderate) were included in the group with good outcome, whereas patients with a score greater than or equal to 4 points (severe sequelae, extremely severe sequelae, or death) were included in the group with poor outcome.

\section{Laboratory examination}

The results of blood and brain crest fluid examination on the first day of admission were recorded in detail. Blood tests included blood routine, biochemical tests. CSF was examined for intracranial pressure, cells, protein, sugar, chloride. The presence of JEV was detected in all serum and CSF samples by IGM capture ELISA.

\section{Radiological examination}

All of the 9 patients underwent brain MR scans upon admission. All MR scans were performed on a 3.0-T scanner using standard protocols. The following modalities were included: $\mathrm{T} 1, \mathrm{~T} 2$, fluid-attenuated inversion recovery (FLAIR), and DWI sequences. Nine patients were examined by CT at the early stage of JE infection. The data related to lesions from imaging were analyzed, including the unique location of lesion, the symmetry, and the number of lesions.

\section{Statistical analysis}

Statistical analysis was performed using the SPSS 20.0 software. Data which conform to normal distribution and have homogeneous variance were presented as the means $\pm \mathrm{SD}$ and analyzed by using the independent sample $T$ test. The measurement data that do not conform to the normal distribution were presented by the median (quartile) $(m(\mathrm{Q} 25, \mathrm{Q} 75))$, the nonparametric Mann-Whitney test was used to compare the skew distribution data groups, and the counting data are described by the percentage (\%). The component ratios between groups were compared by using Fisher's exact test. $p$ value $<0.05$ was considered statistically significant. The binary logistic regression analysis was not examined due to the small number of cases.

\section{Results}

\section{Demographic characteristics}

\section{Common and unique clinical manifestations and their associated outcome}

Of the initial 19 eligible JE patients, 10 were excluded from the analysis because of the absence of data due to referral or abandonment of treatment. The mean hospital admission time of all patients with JE was 4.00 days (SD, 1.94 days) after 
quick onset, and the mean age was 48.89 years (SD, 15.33 years); 6 patients $(66.7 \%)$ were men; the median GCS score was 5 (range, 3-10) at the time of admission. The initial manifestations of JE patients presenting to the hospital included high fever (100\%), the mean temperature was $39.1{ }^{\circ} \mathrm{C}$ (SD, $\left.0.82{ }^{\circ} \mathrm{C}\right)$; headache $((66.7 \%)$; and vomiting $(11.1 \%)$; status epilepticus was observed in 4 patients $(44.4 \%)$, with the median occurrence time of 5 days (range, 3.25-6 days) after onset, and all patients (100\%) experienced varying degrees of consciousness disorder, with an average occurrence time of 4 days (SD, 2.87 days) after onset, including 3 patient with hypersomnia, 2 cases with light coma, 2 cases with moderate coma, and 2 cases with deep coma. In addition, there are 6 cases $(66.7 \%)$ with quadriplegia, 7 cases $(77.8 \%)$ with dyspnea, 7 cases (77.8\%) with meningeal irritation sign, and 4 cases (44.4\%) with pathological sign (Supple. Tables 1 and 2). In univariate analysis, patients with coma $(25 \%(1 / 4)$ vs $100 \%$ $(5 / 5) ; p=0.048)$ or status epilepticus $(0(0 / 4)$ vs $25 \%(1 / 4) ; p=$ 0.048 ) during the course of JE had a significantly poorer outcome (Table 1).

\section{The changes of WBC, serum IgM, and CSF in severe JE}

Almost all patients developed a transient viremia-like changes. The manifestations were the increase of WBC $(22.2 \%)$, neutrophil (100\%), and monocyte $(11.1 \%)$ and the decrease of lymphocyte (100\%), eosinophils (100\%), and platelet (77.8\%) in peripheral blood. A serum IgM antibody test was performed in 5 cases, and the mean time of positive detection was 8.4 days (SD, 1.52 days) (Supple. Table 1). Serological examination revealed that patients with a higher percentage of neutrophils (76 $\pm 1.9 \%$ vs $85 \pm 4.4 \%$; $p=0.006)$ or a lower percentage of lymphocytes $(15.6 \pm 1.7 \%$ vs $8.9 \pm 1.8 \%$; $p=$ 0.001 ) at admission tend to have a poor outcome (Table 2).

The CSF was clear and colorless in appearance; the pressure increased in 1 case $(11.1 \%)$, with an average of 243.13 $\mathrm{mmH}_{2} \mathrm{O}\left(\mathrm{SD}, 25.49 \mathrm{mmH}_{2} \mathrm{O}\right)$; the leucocyte count increased in 9 cases (100\%); 2 cases fluctuated between 10 and $50 \times$ $10^{6} / \mathrm{L}$, and 7 cases fluctuated between 50 and $500 \times 10^{6} / \mathrm{L}$. The protein in CSF increased in 8 cases $(88.9 \%)$ with an average of $577.89 \mathrm{mg} / \mathrm{L}$ (SD, $153.79 \mathrm{mg} / \mathrm{L}$ ), with 2 cases $(22.2 \%)$ with mild abnormality of glucose and chloride. CSF identification showed positive IgM in 9 cases (100\%) with the mean time of 6.56 days (SD, 2.00 days) (Supple. Table 1). There was no difference in all the CSF parameters between the two groups $(p>0.05)$ (Supple. Table 3$)$.

\section{Characteristics of brain lesions in severe JE patients}

Cranial MRI was carried out in all patients with the mean time of 5.14 days (SD, 2.85 days) after onset. Three cases (42.9\%) demonstrated multiple lesions, of which the thalamus was the most frequently involved (85.7\%). Abnormal signals were also seen in the hippocampus (71.4\%), midbrain (28.6\%), temporal lobe (28.6\%), basal ganglia (14.3\%), and insula (14.3\%). MRI showed long T1 and long T2 signals. DWI and FLAIR sequences showed high signal intensity. Head CT of 9 patients showed only one patient with edema in the left temporal lobe, and the mean test time was 3.33 days (SD, 1.87 days) after onset (Supple. Table 2). There was no significant difference between the two groups in the number or

Table 1 Demographic and clinical characteristics between the different groups of SJE.

\begin{tabular}{|c|c|c|c|c|}
\hline $\begin{array}{l}\text { Clinical parameters } \\
N\end{array}$ & $\begin{array}{l}\text { Average/total } \\
9\end{array}$ & $\begin{array}{l}\text { Good prognosis group } \\
4\end{array}$ & $\begin{array}{l}\text { Poor prognosis group } \\
5\end{array}$ & $p$ value \\
\hline Age (year, $\square x \pm s$ ) & $48.89 \pm 15.33$ & $45.75 \pm 19.24$ & $51.4 \pm 13.2$ & $0.616^{\mathrm{a}}$ \\
\hline Admission time (day, $\square x \pm s$ ) & $4 \pm 1.94$ & $3.75 \pm 2.22$ & $4.2 \pm 1.92$ & $0.754^{\mathrm{a}}$ \\
\hline Temperature $\left({ }^{\circ} \mathrm{C}, \square x \pm s\right)$ & $39 \pm 0.82$ & $38.65 \pm 0.94$ & $39.46 \pm 0.55$ & $0.15^{\mathrm{a}}$ \\
\hline GCS (score, $\square x \pm s$ ) & $5(3,10)$ & $10(6,12.5)$ & $3(3,7)$ & $0.056^{\mathrm{c}}$ \\
\hline Headache (case $(\%)$ ) & $6(66.7)$ & $3(75)$ & $3(60)$ & $1^{\mathrm{b}}$ \\
\hline Quadriplegia (case (\%)) & $6(66.7)$ & $3(75)$ & $3(60)$ & $1^{\mathrm{b}}$ \\
\hline Coma (case $(\%))$ & $6(66.7)$ & $1(25)$ & $5(100)$ & $0.048^{\mathrm{b}}$ \\
\hline Status epilepticus (case (\%)) & $4(44.4)$ & $0(0)$ & $4(80)$ & $0.048^{\mathrm{b}}$ \\
\hline Increased muscle tone (case (\%)) & $3(33.3)$ & $1(25)$ & $2(40)$ & $1^{\mathrm{b}}$ \\
\hline Meningeal stimulation sign (case (\%)) & $7(77.8)$ & $2(50)$ & $5(100)$ & $0.167^{\mathrm{b}}$ \\
\hline Dyspnea (case $(\%))$ & $7(77.8)$ & $2(50)$ & $5(100)$ & $0.167^{\mathrm{b}}$ \\
\hline
\end{tabular}

\footnotetext{
${ }^{a}$ Student's $t$ test

${ }^{\mathrm{b}}$ Fisher's exact test

${ }^{\mathrm{c}}$ Mann-Whitney $U$ tests

Italicized text indicates $p<0.05$
} 
Table 2 Hematology examination items of different groups of SJE

\begin{tabular}{lllll}
\hline $\begin{array}{l}\text { Examination item } \\
N\end{array}$ & $\begin{array}{l}\text { Good prognosis group } \\
4\end{array}$ & $\begin{array}{l}\text { Poor prognosis group } \\
5\end{array}$ & Standard value & $p$ value \\
\hline Blood routine examination & & & \\
White blood cell $\left(* 10^{9} / \mathrm{L}\right)$ & $6.52 \pm 1.59$ & $9.09 \pm 4.48$ & -1.080 & $0.316^{\mathrm{a}}$ \\
Neutrophils $(\%)$ & $76 \pm 1.9$ & $85 \pm 4.4$ & -3.84 & $0.006^{a}$ \\
Neutrophils $\left(* 10^{9} / \mathrm{L}\right)$ & $4.95 \pm 1.18$ & $7.75 \pm 3.93$ & -1.358 & $0.217^{\mathrm{a}}$ \\
Monocytes $(\%)$ & $8.5 \pm 0.9$ & $5.1 \pm 3.0$ & 2.366 & $0.066^{\mathrm{a}}$ \\
Monocytes $\left(* 10^{9} / \mathrm{L}\right)$ & $0.56 \pm 0.19$ & $0.49 \pm 0.35$ & 0.362 & $0.728^{\mathrm{a}}$ \\
Lymphocyte $(\%)$ & $15.6 \pm 1.7$ & $8.9 \pm 1.8$ & 5.610 & $0.001^{a}$ \\
Lymphocyte $\left(* 10^{9} / \mathrm{L}\right)$ & $1.01 \pm 0.25$ & $0.84 \pm 0.32$ & 0.846 & $0.425^{\mathrm{a}}$ \\
Blood biochemical examination & & & & \\
AST $(\mathrm{U} / \mathrm{L})$ & $42.25 \pm 48.37$ & $45.90 \pm 27.87$ & -0.143 & $0.89^{\mathrm{a}}$ \\
ALT $(\mathrm{U} / \mathrm{L})$ & $24.83 \pm 25.12$ & $38.18 \pm 23.41$ & -0.824 & $0.437^{\mathrm{a}}$ \\
Creatinine $(\mu \mathrm{mol} / \mathrm{L})$ & $73.63 \pm 17.87$ & $57.22 \pm 8.86$ & 1.819 & $0.112^{\mathrm{a}}$ \\
Urea $(\mathrm{mmol} / \mathrm{L})$ & $4.49 \pm 1.84$ & $5.68 \pm 3.15$ & -0.668 & $0.526^{\mathrm{a}}$ \\
\hline
\end{tabular}

Italicized text indicates $p<0.05$

${ }^{a}$ Student's test distribution of lesions in the whole brain $(66.7 \%(2 / 3)$ vs $25 \%$ $(1 / 4) ; p>0.05$, Table 3).

\section{Management, outcomes, and sequelae}

All patients were admitted to the intensive care unit (ICU) and treated with symptomatic supportive treatment: antiviral therapy with ribavirin, methylprednisolone combined with gamma globulin to inhibit immunity and fight infection, sodium valproate, phenobarbital, and midazolam to control seizures. Intubation and/or tracheotomy were performed in patients with dyspnea, especially who were difficulty in expelling sputum due to respiratory failure, and received extra ventilator, and lowering cranial pressure, nourishing brain cells, physical cooling, oxygen absorption, maintaining water and electrolyte balance, and myocardial protection.

Of the 9 cases, 2 cases (22.2\%) died of the failure of peripheral circulation and respiration at the acute phase, and 3 cases (42.9\%) left severe sequelae, such as irreversible disability ( $\mathrm{mRS} \geq 4$ scores). In addition, the other sequelae include memory or comprehension disorders, aphasias, delayed response, and paralysis, which accounted for $71.4 \%, 57.1 \%$, $57.1 \%, 42.9 \%$, and $42.9 \%$, respectively (Table 4 ).

\section{Discussion}

JE is a type of viral encephalitis caused by a globular virus with a capsular membrane. It is a critical problem of public health worldwide; however, most of the previous studies on JE have focused on children, and there is limited data on the clinical features and prognostic indicators of the eventual outcome of adult with severe Japanese encephalitis. It was reported that JEV takes mosquitoes as its media and pig can be the intermediate host for JEV [2]. Of note, Linyi is the largest city on the covered area and population in Shandong Province of China, with large rice cultivation areas, developed pig industry, the summer rainstorm, and humid warm climate, which provide the ideal conditions for the breeding of mosquitoes and the spread of JEV. The invasiveness of JEV is related to the
Table 3 MRI findings in brain and its composition ratio

\begin{tabular}{lllll}
\hline Parts & Total $(\%)$ & Good prognosis group $(n=3)$ & Poor prognosis group $(n=4)$ & $p$ value \\
\hline Multilesion $(>2)$ & $3(42.9)$ & $2(66.7)$ & $1(25)$ & $0.486^{\mathrm{a}}$ \\
Thalamus & $6(85.7)$ & $2(66.7)$ & $4(100)$ & $0.429^{\mathrm{a}}$ \\
Hippocampus & $5(71.4)$ & $2(66.7)$ & $3(75)$ & $1^{\mathrm{a}}$ \\
Temporal lobe & $2(28.6)$ & $1(33.3)$ & $1(25)$ & $1^{\mathrm{a}}$ \\
Midbrain & $2(28.6)$ & $1(33.3)$ & $1(25)$ & $1^{\mathrm{a}}$ \\
Basal ganglia & $1(14.3)$ & $0(0)$ & $1(25)$ & $1^{\mathrm{a}}$ \\
Insula & $1(14.3)$ & $1(33.3)$ & $0(0)$ & $0.429^{\mathrm{a}}$ \\
\hline
\end{tabular}

${ }^{\text {a }}$ Fisher's exact test 
Table 4 The sequela in 9 cases with SJE

\begin{tabular}{|c|c|c|c|c|c|c|c|c|}
\hline \multirow[t]{2}{*}{$\mathrm{NO}$} & \multicolumn{2}{|c|}{ Mental symptoms } & \multirow{2}{*}{$\begin{array}{l}\text { Memory or comprehension } \\
\text { disorders }\end{array}$} & \multirow[t]{2}{*}{ Aphasias } & \multirow[t]{2}{*}{ Delayed response } & \multirow[t]{2}{*}{ Paralysis } & \multirow[t]{2}{*}{ Incontinence } & \multirow[t]{2}{*}{ MRS } \\
\hline & Dysphoria & Depressed & & & & & & \\
\hline 1 & - & + & + & + & + & + & - & 5 \\
\hline 2 & - & - & + & - & - & - & - & 1 \\
\hline 3 & + & - & + & - & - & + & - & 4 \\
\hline 4 & - & + & - & - & + & - & - & 2 \\
\hline 5 & N/A & N/A & N/A & N/A & N/A & N/A & N/A & 6 \\
\hline 6 & - & - & - & + & - & - & - & 1 \\
\hline 7 & - & + & - & + & + & - & + & 3 \\
\hline 8 & - & + & + & + & - & + & + & 5 \\
\hline 9 & N/A & N/A & N/A & N/A & N/A & N/A & N/A & 6 \\
\hline$n$ & $5(71.4 \%)$ & $4(57.1 \%)$ & $4(57.1 \%)$ & $3(42.9 \%)$ & $3(42.9 \%)$ & $2(28.6 \%)$ & $4(\geq 4)$ & \\
\hline
\end{tabular}

MRS scoring criteria: "0" no symptoms at all; "1" no significant sequelae despite symptoms, ability to perform all usual duties and activities; "2" mild sequelae, not able to complete all previously available activities, but able to handle personal matters without assistance; "3" moderate sequelae, daily life requires assistance, but walking does not; " 4 " severe sequelae, unable to walk without assistance from others and unable to fulfill their own physical needs; " 5 " extremely severe sequelae, bedridden, incontinent, requiring constant care and attention; "6" death

$N / A$, no data

difference of individual susceptibility, but in recent years, the incidence of adult Japanese encephalitis has gradually increased. On the one hand, with the overall incidence of JE decreasing, the chance of natural cryptogenic infection has decreased, leading to a gradual decline in vaccine-induced antibody levels; on the other hand, adults have a higher risk of infection because of the growing social mobility. Studies have shown that enhanced vaccination could provide $96.8 \%$ protection against JE [9]. Therefore, preventive vaccination with live attenuated vaccine and enhanced post-immunization testing can be better at preventing the occurrence of JE.

The disease progresses through prodromal, encephalitic, and convalescent stages. The prodromal stage of JE is characterized by the sudden onset of fever, headache, nausea, and vomiting. The encephalitic stage then begins after 3 days, which typically manifests with dyskinesia (Parkinson's syndrome can be seen in 20 to $60 \%$ of patients), decerebrate rigidity, epilepsy, and neurological deficit [10]. Apart from typical presentations, rare cases which include acute transverse myelitis, cerebral venous thrombosis, and acute flaccid paralysis have also been reported [10-12]. In addition, neurological deficit such as hemiplegia is not an unusual phenomenon in the encephalitis stage, but as an initial manifestation, it is rare. A 52-year-old male in the current study with the initial presentation of left hemiplegia and fever developed lethargy after 2 days of hemiplegia. Anti-JEVspecific IgM had tested positive in the CSF and serum samples in 5 days, and the brain imaging revealed the high signal in the right basal ganglion, thalamus, and cerebral peduncle on DWI. It is possible that the lesions affected by the virus concentrated on the cortical spinal tracts, which occurred prior to the whole brain cells involved due to the over-activation of innate and adaptive immune cells.
In this study, all patients were admitted with high fever and experienced different degrees of consciousness disorders. The damage of thalamus (wake center) and extensive cortex can cause different degrees of disturbance of consciousness, which confirms that the degree of consciousness disorders is correlated with the severity of brain tissue injury. Besides, decreased consciousness and duration were associated with recurrent seizures, which were considered to be prediction factors for death [13]. This study also showed that coma ( $p$ $<0.05$ ) may be associated with poorer outcome of severe JE.

It was been reported that the damage of hippocampus is also a very important cause of epileptic seizures, which enhanced neuronal excitability and decreased levels of inhibitory neurotransmitter dynorphin, leading to seizures [14]. Our data showed that the hippocampus was involved in $50 \%$ of the 4 patients with epileptic persistence, which is in agreement with the literature. Epilepsy attack and brain tissue injury are cause and effect; brain tissue injury can lead to seizures, and seizures can cause neuronal damage, which repeated injury aggravates the damage to brain parenchyma. Studies have shown that seizures often occur in the early stages of JE, and prehospital seizures do not increase mortality, but patients with status epilepticus are more likely to die than patients with selflimited seizures [15]. Moreover, status epilepticus had been shown to be associated with poorer outcomes for JE, which often indicated the increased intracranial pressure [13]. Our data expand beyond the previous findings and show that status epilepticus may be associated with poorer outcomes of severe $\mathrm{JE}$, and only one (11.1\%) patient showed increased intracranial pressure far below the 50\% reported [13]; therefore, we consider that status epilepticus of severe JE may be associated with the damage to the extensive brain and the hippocampus. 
In addition, some patients with systemic inflammation can lead to organ damage, lung infection, multiple organ failure, upper gastrointestinal hemorrhage, brain hernia, and other complications [16]. Dyspnea and respiratory failure are common clinical symptoms in severe patients which usually occur during the encephalitic phase of JE [16]. In this cohort in Linyi, China, 2 patients $(22.2 \%)$ died of respiratory failure; the time of death was 7 days and 13 days after admission, which were longer than reported, and our data demonstrated that the incidence of dyspnea was $77.8 \%$, with no significant correlation between dyspnea and the outcome of patients $(p>0.05)$.

In this study, all hospitalized patients with JE were statistically analyzed for JE antibody, blood routine, liver and kidney function, cerebrospinal fluid, and other laboratory tests. Laboratory diagnosis of JEV is by IgM capture ELISA with high sensitivity and specificity. The positivity of JEV antibody (IgM) in the CSF reflects central nervous system JEV infection, which is usually detected in serum about 7 days after infection in $90 \%$ of patients, while in CSF tests, it can be positive as early as 1 day after onset $[17,18]$. During JEV infection, an increased neutrophil in the peripheral blood has been observed, which could degrade the virus by activating NADPH to release superoxide anion, it forms an early line of defense $[2,19]$. Our data showed that patients with a higher percentage of neutrophils and a lower percentage of lymphocytes at admission tend to have a poorer outcome, which means that the balance between antiviral and inflammatory responses may be an important factor in outcome.

In this study, cerebrospinal fluid examination showed that the leucocyte count in CSF of patients with JE increased in different degrees, mainly in mild and moderate degrees $\left(<500 \times 10^{6} / \mathrm{L}\right)$. The majority of patients with JE (88.9\%) had a slight increase in the level of CSF protein, which did not exceed $1.0 \mathrm{~g} / \mathrm{L}$. The glucose and chloride levels were normal to low (22.2\%), which were the same as those reported in literature [20]. Further analysis showed that there was no significant correlation between CSF parameters and poor outcome $(p>0.05)$, considering that the organ function damage caused by viremia was transient and reversible. Timely administration of the drug can effectively improve, and patients have different hospital admission time after onset, thus resulting in bias in the collection of blood samples and brain crest fluid; in addition, the sample size is small; the relationship between laboratory findings and the outcome of patients can be further studied by enlarging the sample size.

JEV mainly affects the cerebral parenchyma [21]. However, univariate analysis did not show a correlation between the radiological changes and the outcome in the current study; among the 9 patients, 6 cases $(85.7 \%)$ suffered from thalamic involvement. Bilateral diffuse thalamic hyperintensities have been shown in 4 cases. One of our unanticipated findings was that the hippocampal is the second most common lesion (71.4\%), where the lesions often involved the tail and body, and 2 patients $(40 \%)$ are showing the amygdala and the uncinate involvement, while the secondary lesion of adults reported is often the midbrain [8]. Besides, temporal lobe involvement was seen in 2 cases $(28.6 \%)$ in our study. Temporal lobe involvement can often lead to secondary epilepsy patients and language dysfunction, and we discovered that cognitive function in patients with temporal lobe involvement improved quite slowly, often leaving aphasias (50\%). In addition, it often needs to be distinguished from herpes simplex encephalitis when the temporal lobe is involved, but the latter often invades the limbic system, especially the unilateral medial temporal lobe, which is also seen in the insula and cingulate gyrus, while involvement of both brain stem and basal ganglia is rare [22, 23].

At present, there is no standard to evaluate the outcome of JE patients at home and abroad $[24,25]$. We found that even the clinical status of many JE survivors has improved when discharged, the patients that suffered from severe irreversible disability ( $\mathrm{mRS} \geq 4)$ in the later stage of rehabilitation could still reach $42.9 \%$. In addition, we found out that the memory impairment of adults with severe JE (57.1\%) is more serious than reported, which may be related to the increase of hippocampus injury mentioned above, which is critical to the longterm memory. There are extensive neural connections between hippocampus and the anterior thalamic nucleus [26], which increased the memory damage. All the patients with memory impairment in this study showed damage to the thalamus and hippocampus that confirmed the opinions above. Interestingly, a patient in the current study has recovered from score 4 to score 1 after half a year of rehabilitation exercise (language, movement, psychology, etc.), which may be related to prompt rehabilitation, the economic condition of the family, and the understanding of the patient and his family. Therefore, it is extremely important to carry out scientific and effective rehabilitation in time during the recovery period.

\section{Conclusion}

Collectively, our data suggested a correlation between the fatal outcome and the degree of inflammatory responses. Damage to the hippocampus may be associated with status epilepticus and indicate long-lasting memory problems, especially when combined with thalamus damage. Coma and status epilepticus in adults than that of children may be associated with poorer outcome of severe JE. Early supportive treatment may limit the progression of JE and significantly improve the outcome. Due to the limitation of the retrospective design and the current small-cohort study, a multicenter study with larger populations and a more precise prospective study are warranted to further validate our findings.

Authors' contribution SQ and XZX finished most of the experiment. HF, LZC, SXJ, and ZFC helped with the data analysis. LD and CFY designed the study and wrote the manuscript. 
Funding This work was supported by grants from the Shandong Provincial Natural Science Foundation, China (ZR2016HB27).

Data availability All the original data and materials are available from the corresponding author.

\section{Compliance with ethical standards}

Conflict of interest The authors declare that they have no conflict of interest.

Ethics approval and consent to participate Informed consent was obtained from all the participants. This study was approved by the ethics committee of Linyi People's Hospital.

Consent for publication All authors agreed with the publications.

\section{References}

1. Ghoshal A, Das S, Ghosh S, Mishra MK, Sharma V, Koli P, Sen E, Basu A (2007) Proinflammatory mediators released by activated microglia induces neuronal death in Japanese encephalitis. Glia 55(5):483-496. https://doi.org/10.1002/glia.20474

2. Lannes N, Summerfield A, Filgueira L (2017) Regulation of inflammation in Japanese encephalitis. J Neuroinflammation 14(1): 158. https://doi.org/10.1186/s12974-017-0931-5

3. Wang H, Liang G (2015) Epidemiology of Japanese encephalitis: past, present, and future prospects. Ther Clin Risk Manag 11:435448. https://doi.org/10.2147/TCRM.S51168

4. Connor B, Bunn WB (2017) The changing epidemiology of Japanese encephalitis and New data: the implications for New recommendations for Japanese encephalitis vaccine. Trop Dis Travel Med Vaccines 3:14. https://doi.org/10.1186/s40794-017-0057-x

5. Campbell GL, Hills SL, Fischer M, Jacobson JA, Hoke CH, Hombach JM, Marfin AA, Solomon T, Tsai TF, Tsu VD, Ginsburg AS (2011) Estimated global incidence of Japanese encephalitis: a systematic review. Bull World Health Organ 89(10): 766-774, 774A-774E. https://doi.org/10.2471/BLT.10.085233

6. Kumar R, Mathur A, Kumar A, Sharma S, Chakraborty S, Chaturvedi UC (1990) Clinical features \& prognostic indicators of Japanese encephalitis in children in Lucknow (India). Indian J Med Res 91:321-327

7. Organization WH (2008) WHO-recommended standards for surveillance of selected vaccine preventable diseases. World Health Organization

8. Basumatary LJ, Raja D, Bhuyan D, Das M, Goswami M, Kayal AK (2013) Clinical and radiological spectrum of Japanese encephalitis. J Neurol Sci 325(1-2):15-21. https://doi.org/10.1016/j.jns.2012.11. 007

9. Chen KM, Tsai HC, Sy CL, Lee SS, Liu YC, Wann SR, Wang YH, Mai MH, Chen JK, Wu KS, Chen YJ, Chen YS (2009) Clinical manifestations of Japanese encephalitis in southern Taiwan. J Microbiol Immunol Infect 42(4):296-302

10. Verma R, Praharaj HN, Patil TB (2012) Giri P (2012) Acute transverse myelitis following Japanese encephalitis viral infection: an uncommon complication of a common disease. BMJ Case Rep 2012:bcr2012007094. https://doi.org/10.1136/bcr-2012-007094

11. Mokkappan S, Basheer A, Iqbal N (2015) Chidambaram S (2015) Bilateral thalamic bleed and cerebral venous sinus thrombosis in Japanese encephalitis. BMJ Case Rep 2015:bcr2014207957. https://doi.org/10.1136/bcr-2014-207957
12. Chung CC, Lee SS, Chen YS, Tsai HC, Wann SR, Kao CH, Liu YC (2007) Acute flaccid paralysis as an unusual presenting symptom of Japanese encephalitis: a case report and review of the literature. Infection 35(1):30-32. https://doi.org/10.1007/s15010-0076038-7

13. Solomon T, Dung NM, Kneen R, Thaole TT, Gainsborough M, Nisalak A, Day NP, Kirkham FJ, Vaughn DW, Smith S, White NJ (2002) Seizures and raised intracranial pressure in Vietnamese patients with Japanese encephalitis. Brain 125(Pt 5):1084-1093. https://doi.org/10.1093/brain/awfl 16

14. Solbrig MV, Adrian R, Chang DY, Perng GC (2006) Viral risk factor for seizures: pathobiology of dynorphin in herpes simplex viral (HSV-1) seizures in an animal model. Neurobiol Dis 23(3): 612-620. https://doi.org/10.1016/j.nbd.2006.05.014

15. Solomon T, Mallewa M (2001) Dengue and other emerging flaviviruses. J Infect 42(2):104-115. https://doi.org/10.1053/jinf. 2001.0802

16. Sarkari NB, Thacker AK, Barthwal SP, Mishra VK, Prapann S, Srivastava D, Sarkari M (2012) Japanese encephalitis (JE). Part I: clinical profile of 1,282 adult acute cases of four epidemics. J Neurol 259(1):47-57. https://doi.org/10.1007/s00415-011-6118-6

17. Do LP, Bui TM, Hasebe F, Morita K, Phan NT (2015) Molecular epidemiology of Japanese encephalitis in northern Vietnam, 19642011: genotype replacement. Virol J 12:51. https://doi.org/10.1186/ s12985-015-0278-4

18. Japanese encephalitis vaccines (2006). Wkly Epidemiol Rec 81 (34/35):331-340

19. Srivastava S, Khanna N, Saxena SK, Singh A, Mathur A, Dhole TN (1999) Degradation of Japanese encephalitis virus by neutrophils. Int J Exp Pathol 80(1):17-24. https://doi.org/10.1046/j.1365-2613. 1999.00092.x

20. Kant Upadhyay R (2013) Biomarkers in Japanese encephalitis: a review. Biomed Res Int 2013:591290-591224. https://doi.org/10. 1155/2013/591290

21. Bertrand A, Leclercq D, Martinez-Almoyna L, Girard N, Stahl JP, De-Broucker T (2017) MR imaging of adult acute infectious encephalitis. Med Mal Infect 47(3):195-205. https://doi.org/10.1016/ j.medmal.2017.01.002

22. Handique SK, Das RR, Barman K, Medhi N, Saharia B, Saikia P, Ahmed SA (2006) Temporal lobe involvement in Japanese encephalitis: problems in differential diagnosis. AJNR Am J Neuroradiol 27(5):1027-1031

23. Wei Y, Guan H (2020) Pitfalls in the diagnosis of herpes simplex virus-1 encephalitis: case report. Neurol Sci. https://doi.org/10. 1007/s10072-020-04765-Z

24. Sarkari NB, Thacker AK, Barthwal SP, Mishra VK, Prapann S, Srivastava D, Sarkari M (2012) Japanese encephalitis (JE) part II: 14 years' follow-up of survivors. J Neurol 259(1):58-69. https:// doi.org/10.1007/s00415-011-6131-9

25. Ooi MH, Lewthwaite P, Lai BF, Mohan A, Clear D, Lim L, Krishnan S, Preston T, Chieng CH, Tio PH, Wong SC, Cardosa J, Solomon T (2008) The epidemiology, clinical features, and longterm prognosis of Japanese encephalitis in central Sarawak, Malaysia, 1997-2005. Clin Infect Dis 47(4):458-468. https://doi. org/10.1086/590008

26. Fischl B, Salat DH, Busa E, Albert M, Dieterich M, Haselgrove C, van der Kouwe A, Killiany R, Kennedy D, Klaveness S, Montillo A, Makris N, Rosen B, Dale AM (2002) Whole brain segmentation: automated labeling of neuroanatomical structures in the human brain. Neuron 33(3):341-355. https://doi.org/10.1016/s08966273(02)00569-x

Publisher's note Springer Nature remains neutral with regard to jurisdictional claims in published maps and institutional affiliations. 